\title{
Bridge-Output-to-Frequency Converter for Smart Thermal Air-Flow Sensors
}

\author{
Gert J. A. van Dijk and Johan H. Huijsing
}

\begin{abstract}
A fully integrated bridge-output-to-frequency converter has been realized as signal-conditioning circuitry for a onechip smart thermal mass-fiow sensor. The frequency converter reads out four separate resistor bridges of the air-flow sensor in order to realize high sensitivity and directional flow measurement over the full angle range of $360^{\circ}$. The converter selects the sensor bridges individually by means of a microprocessorcontrolled analog multiplexer. The flow velocity and direction are also calculated by the microprocessor. The relative measurement cancels all first-order errors. The center frequency is $10 \mathrm{kHz}$ and the sensitivity $1 \mathrm{~Hz} /(\mu \mathrm{V} / \mathrm{V})$. A bridge-ofiset of $10 \mathrm{mV} / \mathrm{V}$ is allowed, and the linearity error is $0.1 \%$.
\end{abstract}

\section{INTRODUCTION}

$\mathrm{N}$ THIS section the thermal air-flow sensor and the smart air-flow sensor concept will be introduced. The bridgeoutput-to-frequency converter will be investigated, and some measurement results will be shown in Section II.

\section{A. Direction-Sensitive Thermal Air-Flow Sensor}

On-chip thermal air-flow measurement is mostly based on measurement of temperature gradients on a heated chip This general principle of the thermal mass-flow and more particularly air-flow measurement is shown in Figs. 1 and 2. If an embedded chip is heated to a constant temperature above ambient temperature by a central heater element, heat transfer to the air flow is not distributed uniformly over its surface. The surface upstream is being cooled more strongly than downstream. Due to finite thermal conductivity of the chip, differential cooling results in a temperature gradient, $\Delta T$, in the chip in the direction of the air flow.

The temperature difference, $\Delta T$, is a measure of onedimensional flow. Temperature distribution of the heated chip in the direction of flow in the cross section of the sensor is shown in Fig. 2. The origin is placed in the center of the chip. The $X$-axis represents the position on the chip in the direction of flow, and the $T$-axis, the absolute temperature on the surface of the chip, where $T a$ represents the ambient reference temperature. The symmetrical dashed curve shows the temperature distribution if there is no flow across the surface yielding the highest temperature is the temperature above the center of the chip, $T c$. The asymmetric continuous curve shows the temperature distribution under influence of the flow. It also shows the temperature difference $\Delta T$ that is generated by the flow between both ends of the heated chip.

Manuscript received October 6, 1993; revsied June 23, 1994.

The authors are with the Delft University of Technology, 2628 CD Delft, The Netherlands.

IEEE Log Number 9411534

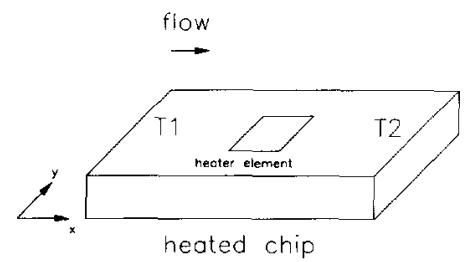

Fig. 1. Temperature difference caused by flow over the surface of a heated chip.

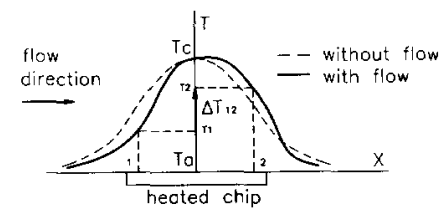

Fig. 2. Influence of the flow on the temperature distribution.

The square root of the flow is proportional to this temperature difference, $\Delta T$, on the chip divided by the temperature difference, $T c a$, between the center of the chip and the ambient [1], according to (1)

$$
\sqrt{\text { Flow }} \propto\left(\frac{\Delta T}{T c a}\right) .
$$

One method of measuring this flow-induced temperature gradient is to apply temperature-sensitive resistors.

Conventional thermal air-flow measurement, where temperature-sensitive resistors are connected in a Wheatstone bridge, [2] has some major disadvantages. Fig. 3 shows an example of such an approach applied in a hot-wire anemometer. All resistors are heated above the ambient temperature by passing an electric current through them. They will be simultaneously cooled by the passing air flow. If the sensor is placed in an air flow coming from the left as indicated by the arrow, the left side of the chip is cooled more than the right side. Therefore, resistor $R 1$ is cooled more than resistor $R 4$, while resistors $R 2$ and $R 3$ have the same temperature. Due to the temperature coefficient of the resistors, the voltage on point $c$ will increase. The voltage on point $d$ will also increase, but to a lesser extent. This voltage difference $V c d$ is a measure for the flow velocity. A negative voltage output is obtained if the flow comes from above or below. However, if the flow direction is reversed the same output is obtained.

The disadvantages of this method for direction-sensitive thermal air-flow measurement are as follows. First of all the 

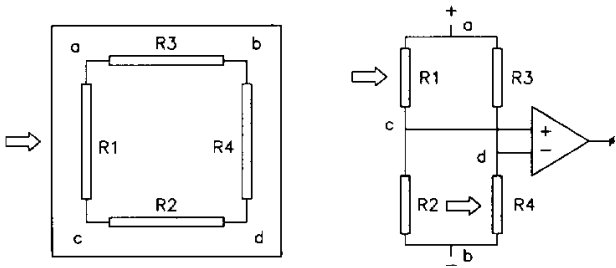

Fig. 3. Thermal air-flow measurement by means of Wheatstone bridge

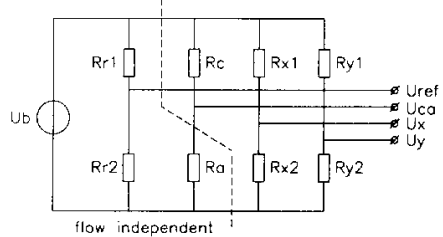

Fig. 4. Electrical sensor circuit.

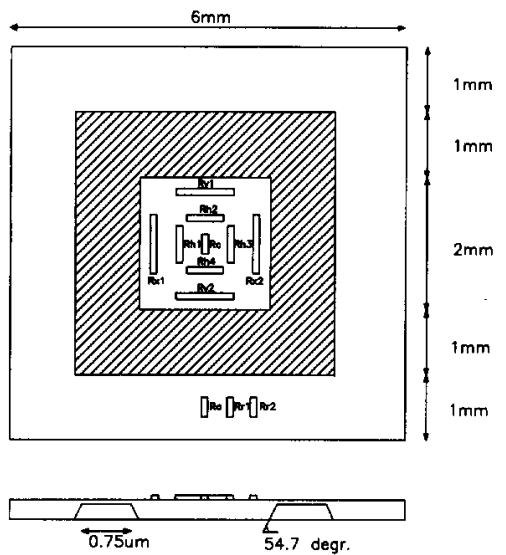

Fig. 5. Flow sensor, top and side views.

measuring principle is based on a second-order effect which deteriorates the sensitivity, and secondly the directional flow sensitivity repeats in cycles of $90^{\circ}$ rotation. For this reason another measuring principle is presented which is based on a first-order effect instead and by which flow velocity and direction over an angle of $360^{\circ}$ can be determined.

This two-dimensional air-flow measurement is realized by equipping the sensor with two separate bridges. Each of these bridges is arranged in such a way that it is sensitive to one flow vector component in a first-order effect. The two bridges together measure two perpendicular flow vectors in order to realize directional flow sensitivity over the full range of $360^{\circ}$ [3]. The electrical circuit of the air-flow sensor is shown in Fig. 4; and top and side views of the sensor are shown in Fig. 5.

The electrical circuit shows a resistor bridge with four separate branches. The branch on the left is a reference branch which has been placed outside the heated air flow. This branch
TABLE I

\begin{tabular}{ll}
\multicolumn{2}{c}{$R x 1,2=R y 1,2=R r 1,2=R c=R a=1 \mathrm{k} \Omega$} \\
$R o=250 \mathrm{k} \Omega ; C o=100 \mathrm{pF} ; R t=5 \mathrm{k} \Omega ; U b s=2 \mathrm{~V}$ \\
\hline Supply voltage & $5 \mathrm{~V}$ \\
Center frequency & $10 \mathrm{kHz}$ \\
Sensitivity & $1 \mathrm{~Hz}(\mu \mathrm{V} / \mathrm{V})$ \\
Nonlinearity & $0.1 \%{ }^{*}$ \\
Bridge supply current & $6 \mathrm{~mA}$ \\
Total supply current & $8 \mathrm{~mA}$ \\
\hline
\end{tabular}

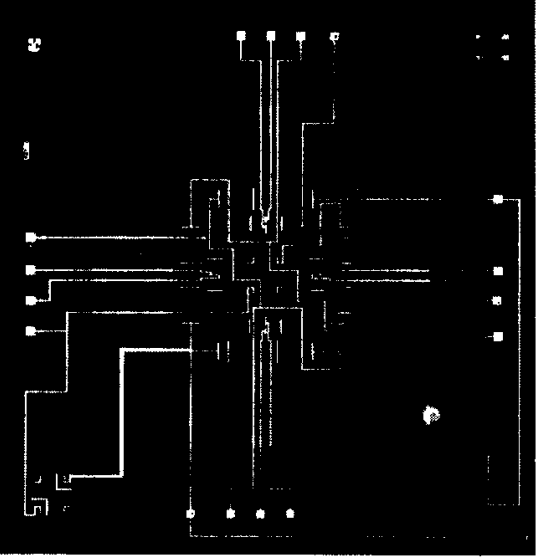

Fig. 6. Photomicrograph of the sensor chip.

only supplies a reference voltage. All resistors to the right of the dashed line are sensitive to temperature differences in the air flow.

Fig. 5 shows that an island has been thermally isolated from the rim of the chip and the ambient by removing most of the silicon to form the thinned ring represented by the hatched area. Heating resistors $R h 1-4$ heat the island to about $10^{\circ} \mathrm{C}$ above the ambient temperature. Sensor bridge branches $R x 1$, $R x 2$ and $R y 1, R y 2$ together with the reference branch $R r 1$, $\operatorname{Rr} 2$ measure the flow-related temperature deviation of the island in two directions. Bridge branch Rca measures the center-to-ambient temperature which is needed to determine the flow. As mentioned above, reference bridge branch $R r 1,2$ and $R a$ are not significantly affected by the flow. See details in Table I.

The flow sensor has been processed in a $3 \mathrm{GHz}$ bipolar process in DIMES (Delft Institute of Microelectronics and Submicron technology). The chip size is $6 \times 6 \mathrm{~mm}$. The thermal isolation area is about $4 \mu \mathrm{m}$ thick (the epilayer). A photograph of the sensor chip is shown in Fig. 6. Experimental results of the flow-sensor measurement are not covered here. For typical behavior of this kind of sensors see [4].

\section{B. Smart Direction-Sensitive Thermal Air-Flow Sensor}

In order to realize a "smart" thermal air-flow sensor, the sensor as well as the signal-conditioning electronics should be implemented on a single chip, as shown in Fig. 7, and be able to present a standardized (digital) output signal to 


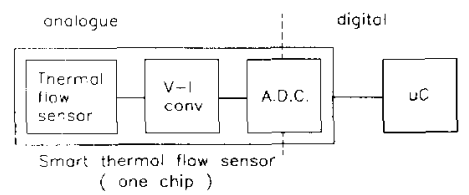

Fig. 7. Combination of sensor and signal conditioning in a smart thermal flow sensor.

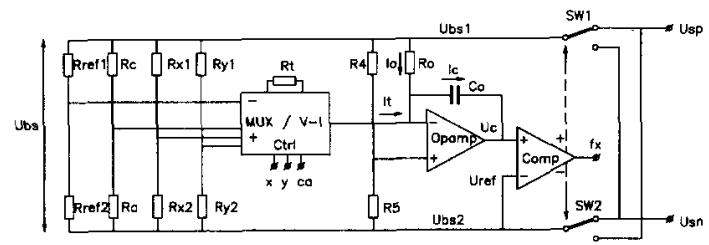

Fig. 8. Basic schematic of the fully integrated converter.

a microcomputer, which processes the data derived from the sensor. The advantages of this approach are that the total system will gain noise and interference immunity, produce standardized output signals, and will be more economical in mass production.

Since the sensor requires most of the chip area, the signalconditioning circuit is restricted to a small part of the chip surface. Furthermore, the electronic circuit has to be fabricated in the same process as the sensor, which rules out the conventional A.D. converters because they require a large chip area. In the application presented here, a frequency converte is used in order to realize an intermediate step towards $A D$ conversion with a circuit that does meet the above-mentioned requirements.

Existing accurate bridge-output-to-frequency converters, where the frequency depends on accurate external $R C$ constant-determining components, cannot be applied directly in a fully integrated frequency converter due to the inaccuracy of the integrated components. To avoid external components a relative instead of an absolute measurement is to be performed by using a measuring and a reference circuit.

This relative measurement cannot be realized by placing two converters on the same sensor chip as this will cause interference and frequency-locking effects. An approach to prevent locking effects and to still be able to read out different bridges, is the application of one frequency converter and a multiplexer, which will be described now.

\section{BRIDGE-OUTPUT-TO-FREQUENCY CONYERTER}

\section{A. Principle of Operation}

The basic circuit of the frequency converter based on this approach, is shown in Fig. 8. On the left-hand side, the different branches of the sensor bridges are connected to a $V-I$ converter in which the positive input contains a multiplexer and the output current is presented to a relaxation oscillator (located at the right-hand side of the diagram).

The relaxation oscillator output frequency is not sensitive to the influence of offset and drift of the input amplifier on

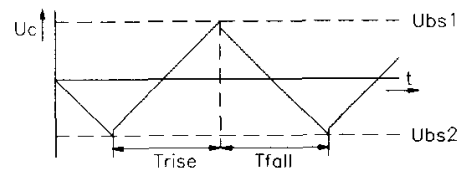

Fig. 9. Integrator output signal.

the bridge-output voltage because the bridge is driven with an alternating voltage [5]. Offset and drift components of the first half-period are opposite of those in the second halfperiod, and therefore the average is zero. The need for external components is eliminated by the relative measuring method.

The frequency output can be connected to a microcontroller which counts the number of pulses per unit time. The bridge imbalance can be determined by calculation of the frequencyshift to center-frequency ratio.

The oscillator consists of an Operational Amplifier (OpA), a comparator and electronic switches. The center frequency, fo, is determined by the integrator capacitor, $C o$, and resistor, $R o$.

Operation can be described as follows. If the bridge is perfectly balanced and the offset of the $V-I$ converter is not taken into account, the output current, $I_{t}$, of the $V-I$ converter is zero. In this situation capacitor, $C o$, is only charged by a current $I c$, which is derived from resistor $R o$ and equals $\frac{1}{2}(U b s / R o)$. Assuming that $U b s 1$ is positive with respect to $U b s 2$, current $I o$ will charge $C o$ in such a way that the output voltage, $U c$, of the integrator decreases until the low reference level of $U b s 2$ is reached (see Fig. 9). At that moment the comparator causes switches $S w 1$ and $S w 2$ to change polarity of the bridge supply voltage $U b s$ as well as the capacitor charge current $I c$. The capacitor $C_{0}$ now discharges, and the integrator output voltage $U c$ increases. As soon as $U_{c}$ reaches the higher reference level of $U b s 2\left(U_{\text {ref }}\right)$, which is now high, the comparator switches the supply voltage, and the whole cycle starts again. If the sensor bridge is out of balance $I_{c}$ consists of a constant current, $I_{o}$, and an output current, $I_{t}$, of the transconductance amplifier, depending on the bridge voltage of the selected bridge branch. In this way the oscillator frequency is shifted by the bridge imbalance. A constant reference voltage of $\frac{1}{2} U b s$ for the OpA is realized by equal resistors $R 4$ and $R 5$. The small jumps in the integrator output signal (exaggerated in Fig. 9 for clarity), are caused by mismatch of resistors $R 4$ and $R 5$ and voltage drops across the switches. This does not, however, affect the frequency.

The output current $I t$ of the $V-I$ converter, caused by imbalance of the selected transducer bridge, $R x$ for example, is added to current $I o$ and can be described by (2)

$$
I t=\frac{U b s}{R t} \cdot\left(\frac{R x 2}{R x 1+R x 2}-\frac{R r 2}{R r 1+R r 2}\right)=k \cdot \frac{U b s}{R t}
$$

where $k$ is defined as the imbalance of the selected transducer bridge.

Capacitor $C_{o}$ is charged to $U b s$ in a half-period and the entire period is described by

$$
f_{x}=\frac{I_{c}}{2 \cdot U b s \cdot C_{o}}=\frac{1}{4 R_{o} \cdot C_{o}}+k \cdot \frac{1}{2 R_{t} \cdot C_{o}}=f_{o}+\Delta f(3)
$$


where

$f x$ output frequency,

fo center frequency,

$\Delta f$ frequency shift,

Ro center frequency-determining resistor,

$C o$ center frequency-determining capacitor,

$k$ bridge imbalance,

Rt transconductance $V-I$ converter,

Rel $x$ relative frequency shift.

The relative measurement is realized as follows. Firstly, the multiplexer input stage connects the reference bridge to the $V-I$ converter, and a microcomputer measures the center frequency fo. Secondly, one of the measure branches is connected, and the output frequency $f x$ is determined. The microcomputer then calculates Relx as the ratio between the frequency shift and the center frequency. This calculated result, Relx, (a frequency ratio) can be described as

$$
R e l x=\frac{f x-f o}{f o}=2 k \cdot \frac{R o}{R t} .
$$

From this formula it can be seen that Relx only depends on the bridge-imbalance $k$ and a ratio between two resistors. This ratio can be very accurate because of the good matching of resistors in the integration process.

\section{B. Realization}

As mentioned above, the total circuit consists of several subcircuits, which will be described briefly below. The V-I converter with multiplexer input stage will be investigated in more detail, due to its essential role.

1) V-I converter and analog multiplexer: Analog switching of floating voltages is hard to realize in bipolar technology, and therefore a multiplexer has been combined with a balanced $V-I$ converter, consisting of two transconductance amplifiers, according to Fig. 10 The differential output current is a measure for the differential input voltage between one of the measuring voltages $U_{x}, U_{y}$ or $U_{c a}$ and the reference voltage $U_{\text {ref. }}$. One of the inputs of the balanced $V-I$ converter is multiplexed, in order to be able to select one of the branches of the measure bridge. The other input is connected to the reference branch at all times.

A more detailed circuit layout of the balanced $V-I$ converter is shown in Fig. 11. Differential input stages $Q 1-8, Q 17-18$ together with the transistors $Q 9, Q 16$ form the two voltage followers $A 1$ and $A 2$ shown in Fig. 10. $R_{t 1}$ and $R_{t 2}$ determine the transconductance. The output currents from the cascaded CE output stages $Q 9$ and $Q 16$ pass the folded cascades $Q 10$ and $Q 15$ and are added together by a current mirror formed by $Q 11-Q 14$ which realizes differential to single-ended current conversion. The differential input stages $Q 1,2-Q 7,8$ are selected by switching the tail current.

2) Operational amplifier: A $1 \mathrm{GHz}$ operational amplifier, developed at the Electronic Instrumentation Laboratory, was scaled to an operational amplifier with a bandwidth

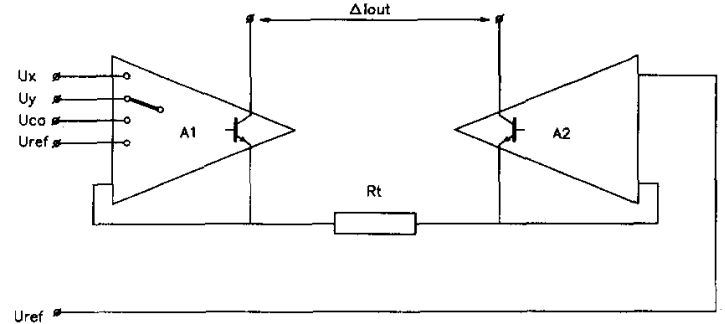

Fig. 10. Principle of balanced $V$-Iconverter with multiplexer input stage.

of $10 \mathrm{MHz}$ and a slew-rate of $16 \mathrm{~V} / \mu \mathrm{s}$ [6]. The linear error caused by this slew-rate limitation is cancelled and will therefore not effect Relx.

3) Comparator and switches: A fast comparator and switches have been chosen ( $<100 \mathrm{~ns})$ in order to prevent nonlinear frequency errors.

\section{Accuracy}

The design criterion is the ability to measure a variation in bridge output of $1 \mu \mathrm{V} / \mathrm{V}$ while a stationary bridge imbalance up to $10,000 \mu \mathrm{V} / \mathrm{V}$ is still allowed. If the chosen sensitivity is $1 \mathrm{~Hz}$ per $1 \mu \mathrm{V} / \mathrm{V}$, then the center frequency has to be $10 \mathrm{kHz}$. The nonlinearity should not exceed $0.1 \%$ in order to match the nonlinearity of the integrated sensor bridge. Because a relative measurement is done, the relative accuracy counts. The following error sources are significant:

1) V-I converter: The main error source of the $V-I$ converter is the input offset which causes a nonlinear second-order error. Errors due to drift and voltage dependence of $R_{t}$ (if polysilicon resistors are applied) are negligible, while linear errors like inaccuracy of $R_{t}$ are cancelled by the measuring method.

Thus remaining errors are:

a) Input offset (second-order error) : If a quad input stage is chosen, the offset will be about $0.5 \mathrm{mV}$. The error will be cancelled in the first order, but a second-order component still remains. This component contributes a relative error of

$$
\frac{\delta R e l_{x}}{R e l_{x}}=8\left(\frac{U_{\text {offs }}}{U b s} \cdot \frac{R_{o}}{R_{t}}\right)^{2}
$$

This error contributes about $0.1 \%$ and may be cancelled if a one-point calibration is performed by the microcomputer.

b) Matching $R_{o}$ and $R_{t}$ : Matching in the integrated process is better than $0.1 \%$.

2) Integrator

a) Input offset: An offset of the operational amplifier of $0.5 \mathrm{mV}$ contributes a relative error of

$$
\frac{\delta \operatorname{Re}_{x}}{\operatorname{Re}_{x}}=8\left(\frac{U_{\text {offs }}}{U b s}\right)^{2}
$$

yielding $5 \times 10^{-7}$ which is negligible with respect to the nonlinearity due to the offset of the $V-I$ converter. 


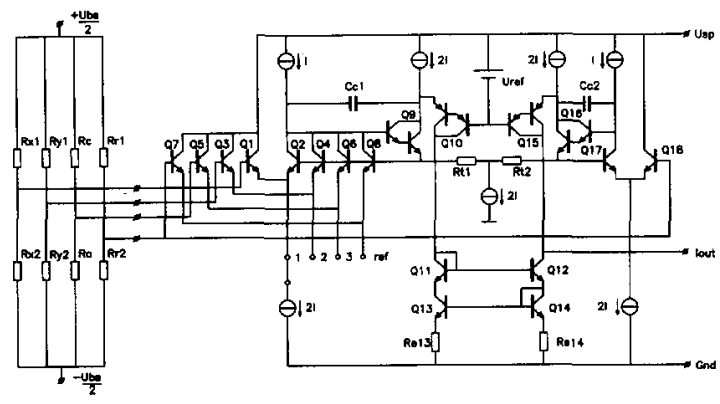

Fig. 11. Balanced V-I converter with multiplexer input stage

b) Slew-rate amplifier: The slew-rate of the amplifier adds a linear error of

$$
\frac{\delta \operatorname{Rel}_{x}}{\operatorname{Rel}_{x}}=\frac{2 f_{x} \cdot U b s}{S r} \approx \frac{2 f_{o} \cdot U b s}{S r}
$$

which amounts to $2.5 \times 10^{-3}$. This is a linear error and is therefore cancelled by the relative measurement.

3) Comparator and switches: The delay time of the comparator and switches contributes a linear error of

$$
\frac{\delta \operatorname{Rel}_{x}}{\operatorname{Rel}_{x}} \approx 4 t d \cdot f_{x} \approx 4 t d \cdot f_{o}
$$

which is cancelled by the relative measurement, resulting in a nonlinearity lower than $0.01 \%$.

Therefore the remaining nonlinear errors can be listed as follows:

$$
\begin{array}{ll}
\text { nonlinearity due to delay time comparator } & 1 \times 10^{-4} \\
\text { second order due to offset V-I converter } & 1 \times 10^{-3} \\
\text { total: } & \approx 1 \times 10^{-3} .
\end{array}
$$

Thus the nonlinearity of the total converter is about 0.1 percent, which equals the nonlinearity of the bridges. Table I shows the simulated specifications of the converter.

This circuit was also processed by DIMES in a standard 3 $\mathrm{GHz}$ bipolar process with a chip size of $2.5 \times 4.5 \mathrm{~mm}$. Fig. 12 shows the layout of the bridge-output-to-frequency converter. A nonlinearity of $0.1 \%$ can be realized if quad cross-coupled input stages are applied.

\section{MEASUREMENT Results}

The measurement results are shown in Figs. 13 and 14. Fig. 13 shows the output frequency of the converter as a function of the bridge imbalance. The area between the two vertical dashed lines shows the range in which the converter is actually used. It can be seen that the measured center frequency did not equal the designed center frequency. This was due to changes in the bipolar processing. While the resistance values were decreased by a factor of two, the center frequency of the practical circuit was doubled. Fig. 14 shows the calculated differential sensitivity $\left(f_{x}-f_{o}\right) / f_{o}$ as a function of the bridge imbalance. Here it can be seen that change of

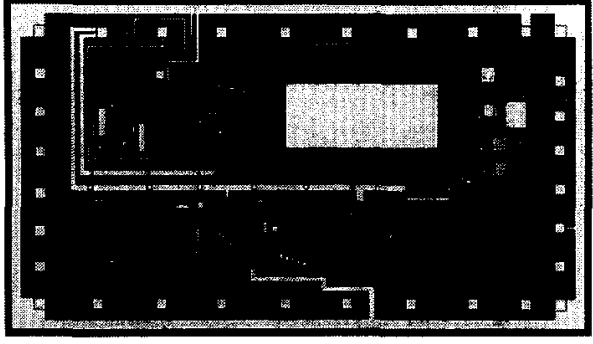

Fig. 12. Photomicrograph of the frequency converter.

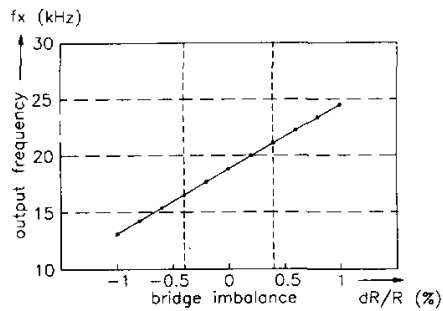

Fig. 13. Output frequency versus bridge imbalance.

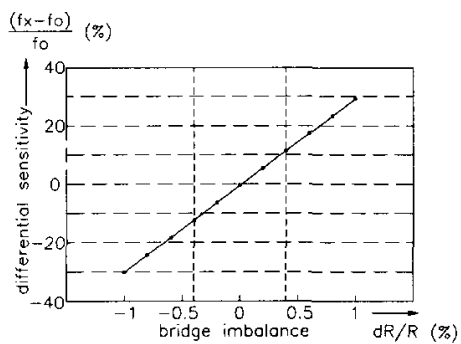

Fig. 14. Differential sensitivity versus bridge imbalance.

the center frequency has no effect on the relative frequency shift $\left(f_{x}-f_{o}\right) / f_{o}$.

\section{CONCLUSION}

An accurate bridge-output-to-frequency converter for smart thermal air-flow sensor has been realized in the following way:

A) By driving the bridge with an alternating voltage at the output frequency the instability caused by offset and drift of input amplifier has been eliminated.

B) Due to the inaccuracy of the integrated RC-constantdetermining components, relative measurement is applied which cancels all linear and most of the absolute errors.

C) Application of a single frequency converter and multiplexer instead of more than one converter on the chip prevents locking and interference problems.

D) A frequency ratio between frequency shift and center frequency represents the airflow. Flow velocity and direction can be calculated from this frequency ratio by a micro-computer which can also select the input bridge branches by means of the multiplexer control input. 


\section{REFERENCES}

[1] H. Schlichting, Boundary Layer Theory. New York: McGraw-Hill, 1979, 7th ed., pp. 265-326.

[2] A. F. P. van Putten, "An integrated silicon double bridge anemometer," Sensors \& Actuators, Elsevier Sequoia S.A., vol. 4, pp. 387-396, 1983.

[3] B. W. van Oudheusden, "Integrated silicon flow-direction sensor," Sensors \& Actuators, Elsevier Sequoia S.A., vol. 16, pp. 109-119, 1989.

[4] , "Silicon thermal flow sensors," Sensors \& Actuators, Elsevier Sequoia S.A., vol. 30, pp. 15-24, 1992.

[5] J. H. Huijsing, G. A. van Rossum, and M. van der Lee, "Twowire bridge-to-frequency converter," IEEE JSSC, vol. SC22, no. 3, pp. 343-349, 1987.

[6] K. J. de Langen and J. H. Huijsing, "1-GHz operational amplifier with multipath nested Miller compensation," Proceedings ISCAS, vol. 5, pp. $517-519,1994$

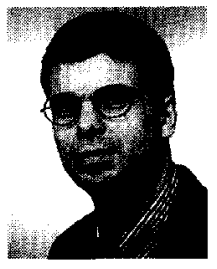

Gert J. A. van Dijk was born in Vlissingen, The Netherlands, on August 14, 1958. He received the B.S. degree in electrotechnical engineering from the Windesheim College, Zwolle, The Netherlands, in 1980.

Currently he is a research assistant at the Electronic Instrumentation Laboratory, Department of Electrical Engineering, of the Delft University of Technology and is involved in research and design of operational amplifiers and integrated smart sensors.

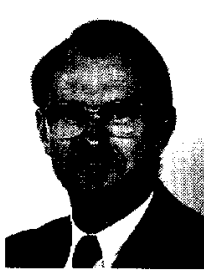

Johan H. Huijsing was born in Bandung, Indonesia, on May 21, 1938. He received the M.Sc. degre in electrical engineering from the Delft University of Technology. Delft, The Netherlands, in 1969 and the Ph.D. degree from this University in 1981 for work on operational amplifiers (Thesis: "Intefor work on operational amplifiers (Thesis: "Inte-
grated Circuits for Accurate Linear Analogue Electric Signal Processing," supervised by Prof. Dr. Ir J. Davidse).

Since 1969 he has been a member of the Research and Teaching Staff of the Electronic Instrumentation Laboratory, Department of Electronic Instrumentation. He teaches courses on Electrical Measurement Techniques, Electronic Instrumentation, Operational Amplifiers and Analog-to-Digital Converters. His field of research is analog circuit design (operational amplifiers, analog multipliers, etc.) and integrated smart sensors (signal conditioning on the sensor chip, frequency and digital converters which incorporate sensors, bus interfaces, etc.).

Dr. Huijsing is the author or co-author of some 100 scientific papers and 15 patents. 